\title{
EPIDEMIOLOGÍA NEONATAL DE LA MIGRACIÓN
}

\author{
MIGRANT NEONATAL EPIDEMIOLOGY
}

\author{
Silvia Reyes Delgado* \\ Maritza Uarac Senn**
}

Rec.: 21-09-2020. Acept.: 19-05-2021. Publ.: 30-06-2021

DOI: http://doi.org/10.29035/ucmaule.60.90

\section{RESUMEN}

La inmigración a nuestro país ha significado cambios epidemiológicos en el Hospital de Linares, motivo de este estudio. Se busca conocer los cambios epidemiológicos de Unidad de Parto (UP) y de Neonatología (UN) de un Hospital de Alta Complejidad. Para la metodología se analizaron registros de pacientes haitianas y sus neonatos entre 2016 y 2018 . Se utilizaron estadígrafos descriptivos y compararon con chi-cuadrado. Se encontró que de un total de 6.911 pacientes, había 81 mujeres haitianas ingresadas a up y de 1.499 neonatos que se hospitalizaron en un, 31 eran haitianos. Se observó un aumento de mujeres haitianas a pesar de la disminución global de los ingresos. Consecuentemente hubo un aumento en las hospitalizaciones de neonatos hijos de madres haitianas, a pesar de una disminución global de hospitalizaciones en la un y en mayor porcentaje en comparación a hijos de mujeres de otras nacionalidades. Los principales diagnósticos de neonatos haitianos fueron PEC severo (25\%), hipoglicemia e hiperbilirrubinemia. Se encontró un aumento en días de estadía en relación a otras nacionalidades. Es llamativo el aumento de ingresos de pacientes haitianas, el alto porcentaje de pacientes PEC severo y la mayor estadía promedio, probablemente debido a barrera idiomática y cultural que dificulta el seguimiento de las indicaciones médicas.

Palabras Clave: haitianas, neonato, Linares, inmigrantes.

Sociedad Chilena de Pediatría, sreyesdelgado@gmail.com https://orcid.org/0000-0001-7584-7968

** Sociedad Chilena de Pediatría, maritzauarac@gmail.com 


\section{ABSTRACT}

Migration to our country has led epidemiologic changes in Hospital de Linares, which motivated this study. The objective is to know the epidemiologic changes in the Maternity Ward en Neonate Unit of a High Complexity. We analyzed the admission of the Haitian women and their offspring throughout 2016 to 2018 . We used descriptive stratigraphy and were compared using chi-squared test. Out of 6911 women that gave birth, we found 81 Haitian women. Out of 1499 neonates that were hospitalized, 31 were of Haitian background. We saw an important increase in the number of Haitian women giving birth even though the general birth rate was decreasing. Subsequently there was an increase in the hospitalization of neonates born to Haitian mothers, even though the general neonate hospitalization rate was decreasing and in a higher percentage when compared to the neonates born to women from other nations. The main diagnosis were severe SCA (25\%). Hypoglycemia and Hyperbilirubin. We observed a higher number of total hospitalized period in newborns of Haitian background compared to other nationalities. It's interesting the increase in Haitian background neonates, the higher percentage of severe SCA and the longer stay due to the language and cultural barrier that influences on the mothers following the medical indications.

Key words: Haitian, neonate, Linares, immigrants

\section{INTRODUCCIÓN}

La migración suele estar en la palestra por las presuntas consecuencias que trae consigo el recibir en un país a nuevos ciudadanos con culturas distintas, patologías desconocidas y exigencias insospechadas al aparato estatal. Se estima que cerca de 200 millones de personas ( 3 \% de la población mundial) viven en un país distinto al de su nacimiento (Instituto Nacional de Estadísticas, 2018). En América Latina y el Caribe hay más de 20 millones de personas que residen fuera de su país natal (Instituto $\mathrm{Na}$ cional de Estadísticas Migración, 2003).

Chile se ha considerado fundamentalmente un país de emigrantes, aunque desde hace algunos años esta tendencia ha ido cambiado. Actualmente es un país receptor de migrantes debido, fundamentalmente, a temas de seguridad social, estabilidad económica y mejores condiciones laborales, comparado con los demás países de la región (Instituto Nacional de Estadísticas, 2018; González et al., 2019). Por estas razones, es uno de los tantos destinos que han elegido los emigrantes de países centro y sudamericanos. La situación según el censo poblacional de 2002 mostró que existía una 
población extranjera de 195.320 habitantes, correspondiendo al 1,3\% de la población total, de los cuales 23.150 personas eran menores de 14 años (Gushulak \& MacPherson, 2004). En el último censo (2017), se evidenció una mayor cantidad de inmigrantes, con un total de 746.465 personas nacidas en el extranjero, que declararon ser residentes habituales en Chile, cifra que representa un $4,4 \%$ del total de la población (Instituto Nacional de Estadísticas, 2018). En el mundo, los migrantes representan un 3,5\% de la población total (International Organization For Migration, 2020). En Latinoamérica, Chile es uno de los países en el cual existe mayor flujo de inmigrantes en comparación a los emigrantes (International Organization For Migration, 2020).

La población extranjera en nuestro país proviene principalmente de América del Sur, siendo los países más frecuentes Perú con un 25,2\%, Colombia 14,1\%, Venezuela $11,1 \%$, Bolivia 9,9\%, Argentina 8,9 \% y Haití 8,4 \% (International Organization For Migration, 2020).

La llegada de inmigrantes haitianos ha significado un esfuerzo para el ámbito de la salud mayor debido a la diferencia cultural y la barrera idiomática. Desde el año 2016, ha aumentado exponencialmente la población haitiana, lo cual se ve reflejado en la cantidad de ingresos en la Unidad de Neonatología de hijos de madres haitianas, tema que analizaremos a continuación.

\section{OBJETIVOS}

Se realiza estudio epidemiológico retrospectivo descriptivo de los ingresos de mujeres haitianas en el Centro de Responsabilidad Médico Quirúrgico Gineco-Obstétrico (CRMQ G-O) y de Recién Nacidos (RN) de madres haitianas hospitalizados en la Unidad de Neonatología (UN) del Hospital de Linares entre los años 2016 al 2018.

\section{METODOLOGÍA}

Se recolectó información de los Libros de Ingreso desde el 1 de enero del 2016 al 31 de diciembre del 2018 de la Unidad de Preparto (Upp) del Hospital de Linares. Se registró número de cédula de identidad (RUT), edad de la madre y nacionalidad. En los casos en que no estaba registrada la nacionalidad, se revisó la Ficha Clínica para lograr conocer dicho dato y en algunos casos, se llamó telefónicamente a las pacientes para preguntar su país de origen. Una vez establecida la nacionalidad de la madre, se confecciono planilla con fecha de nacimiento (FN), nombre y edad de la madre, peso de nacimiento del RN (PN), Apgar al minuto, 3,5 y 10 minutos, edad gestacional (EC) y evaluación del peso al nacer (Milad et al., 2010). Además, se registró la presencia de nacimientos múltiples. 
Reyes, S. \& Uarac, M. (2021). Epidemiología neonatal de la migración. UCMaule, 60, enero-junio, 2021, 90-101. DOI: http://doi.org/10.29035/ucmaule.60.90

Se revisó el Libro de Ingreso de la un y se registró el total de días de hospitalización de todos los ingresos en el periodo estudiado. Se revisó Ficha Clínica (FC) de todos los RN de madres haitianas hospitalizados en la Unidad de Neonatología, registrando: edad de la madre; patología materna, previo y durante el embarazo; número de controles; paridad; necesidad de reanimación al momento del nacimiento; diagnóstico de ingreso y egreso del RN.

Se compararon los resultados según país de origen de la madre, aplicando las pruebas estadísticas de chi-cuadrado y Fisher en los casos de análisis de frecuencia, debido al número de variables comparadas y $t$ de Student en el caso de análisis de promedios de peso y días promedio de estadía.

\section{RESULTADOS}

Se detallan los resultados según las diferentes Unidades Clínicas revisadas.

\section{Unidad de preparto y parto (UPP)}

Al revisar los libros de upp del Hospital de Linares de los años 2016 al 2018, se registró un total de 6.971 nacimientos, 161 son hijos de madres extranjeras $(2,3 \%)$ y 83 de estos últimos, son mujeres haitianas (1,2\%). En la tabla 1 se detallan las características comparadas entre mujeres chilenas, haitianas y de otras nacionalidades.

Tabla 1: Comparación de distintas características entre los ingresos de mujeres chilenas, haitianas y de otras nacionalidades.

\begin{tabular}{|l|c|c|c|c|c|c|c|c|c|c|}
\hline & \multicolumn{3}{|c|}{2016} & \multicolumn{3}{c|}{2017} & \multicolumn{3}{|c|}{2018} & SE \\
\hline & CHILE & HAITI & ON & CHILE & HAITI & ON & CHILE & HAITI & ON & $p$ \\
\hline NACIMIENTOS & 2547 & 1 & 23 & 2259 & 6 & 27 & 2004 & 76 & 28 & \\
\hline GEMELAR & 21 & 0 & 0 & 18 & 0 & 0 & 17 & 2 & 2 & \\
\hline PRETERMINOS & 171 & 0 & 0 & 165 & 0 & 1 & 167 & 4 & 2 & \\
\hline GEG & 302 & 0 & 1 & 286 & 0 & 3 & 214 & 3 & 1 & \\
\hline AEG & 2030 & 0 & 20 & 1791 & 5 & 23 & 1628 & 53 & 20 & \\
\hline PEG & 215 & 1 & 2 & 182 & 1 & 1 & 162 & 20 & 7 & $<0,05$ \\
\hline
\end{tabular}

PEG - pequeño para la edad gestacional; GEG - grande para la edad gestacional; AEG - adecuado para la edad gestacional; SE - estadísticamente significativo; ON - otras nacionalidades; RN - recién nacido.

Fuente: Elaboración propia. 
Reyes, S. \& Uarac, M. (2021). Epidemiología neonatal de la migración. UCMaule, 60, enero-junio, 2021, 90-101. DOI: http://doi.org/10.29035/ucmaule.60.90

En 2016 solo una mujer haitiana ingresó a la upp, observándose un aumento al final del 2018, con 74 mujeres haitianas dando a luz. Al analizar el aumento de número de partos de mujeres extranjeras, se observó que es en base a un aumento en números absolutos de mujeres haitianas y el resto de las nacionalidades se mantuvo estable en el tiempo.

En el periodo estudiado, se vio que, del total de hijos de mujeres extranjeras, cada año aumento el porcentaje de hijos de mujeres haitianas en relación con hijos de mujeres de otras nacionalidades. El 2016, una mujer haitiana de 24 mujeres extranjeras (0,04\%); el año 20176 mujeres haitianas de 33 extranjera, (0,26\%) y el año 2018, 76 haitianas de 104 mujeres extranjeras (3,6\%). En el grafico 1 se visualiza la distribución según nacionalidad de las madres en números absolutos y porcentaje.

Gráfico 1. Procedencia de las madres extranjeras registradas en la UPP del Hospital de Linares -2016 al 2018.

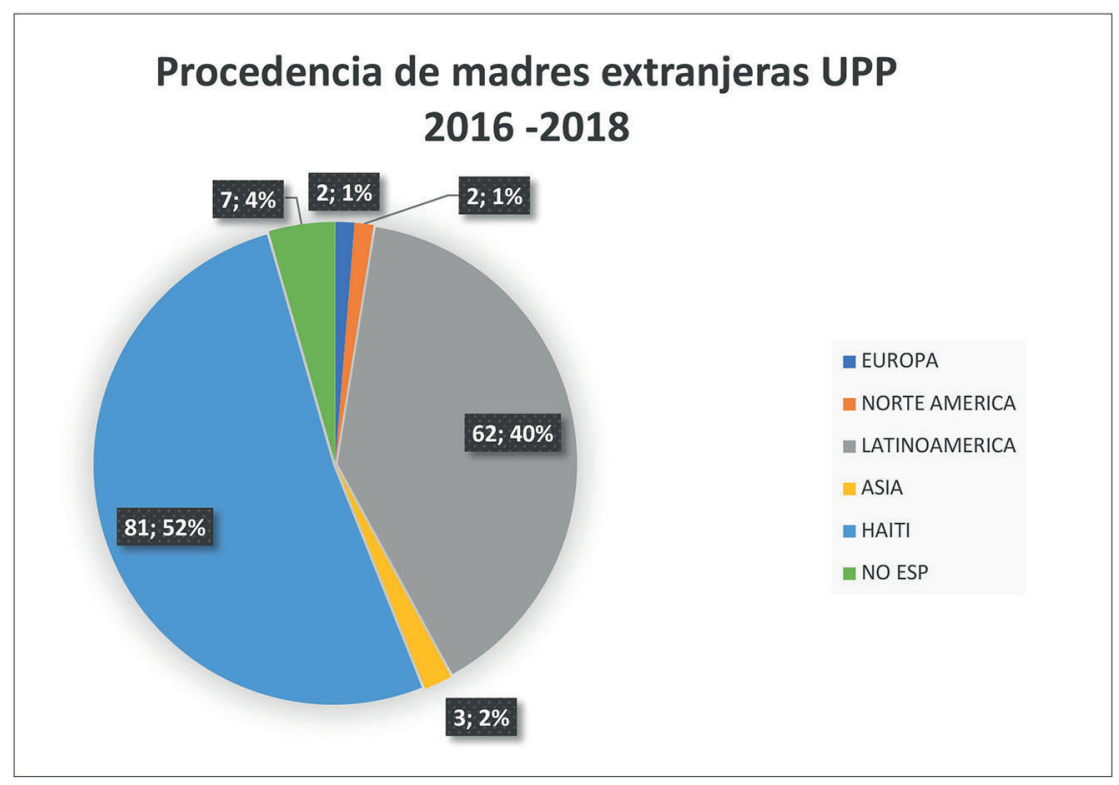

Fuente: Elaboración propia. 
Reyes, S. \& Uarac, M. (2021). Epidemiología neonatal de la migración. UCMaule, 60, enero-junio, 2021, 90-101.

Con respecto a la edad promedio de las mujeres ingresadas a upp en el periodo estudiado, las mujeres chilenas tenían 28,2 años, las mujeres haitianas 28,6 y de otras nacionalidades, 28,8 años.

No hubo embarazos adolescentes en mujeres haitianas y $1,2 \%$ de las embarazadas haitianas tenía sobre los 40 años. En mujeres chilenas, 3,1 \% eran menores de 18 años y $5,1 \%$ eran mayores de 40 años. Las mujeres de otras nacionalidades, ninguna era menor de edad y 5,2\% tenían sobre 40 años. Esta comparación es estadísticamente significativa según prueba de chi-cuadrado $(p<0,025)$.

De los RN de mujeres haitianas, el promedio de peso al nacer fue de 3,086 gramos, los hijos de mujeres de otras nacionalidades fueron de 3,353 gramos y de las mujeres chilenas 3,336 gramos. Estadísticamente significativo según la prueba de $t$ de Student.

En relación al Peso según Edad Gestacional, se observó un mayor porcentaje de Pequeño para la Edad Gestacional (PEC) en el grupo de mujeres haitianas en comparación a chilenas y otras nacionalidades (Gráfico 2), lo cual fue estadísticamente significativo según prueba de chi-cuadrado $(p<0,05)$.

Gráfico 2: Estado Nutricional de los pacientes al nacer, distribuidos según nacionalidad de la madre.

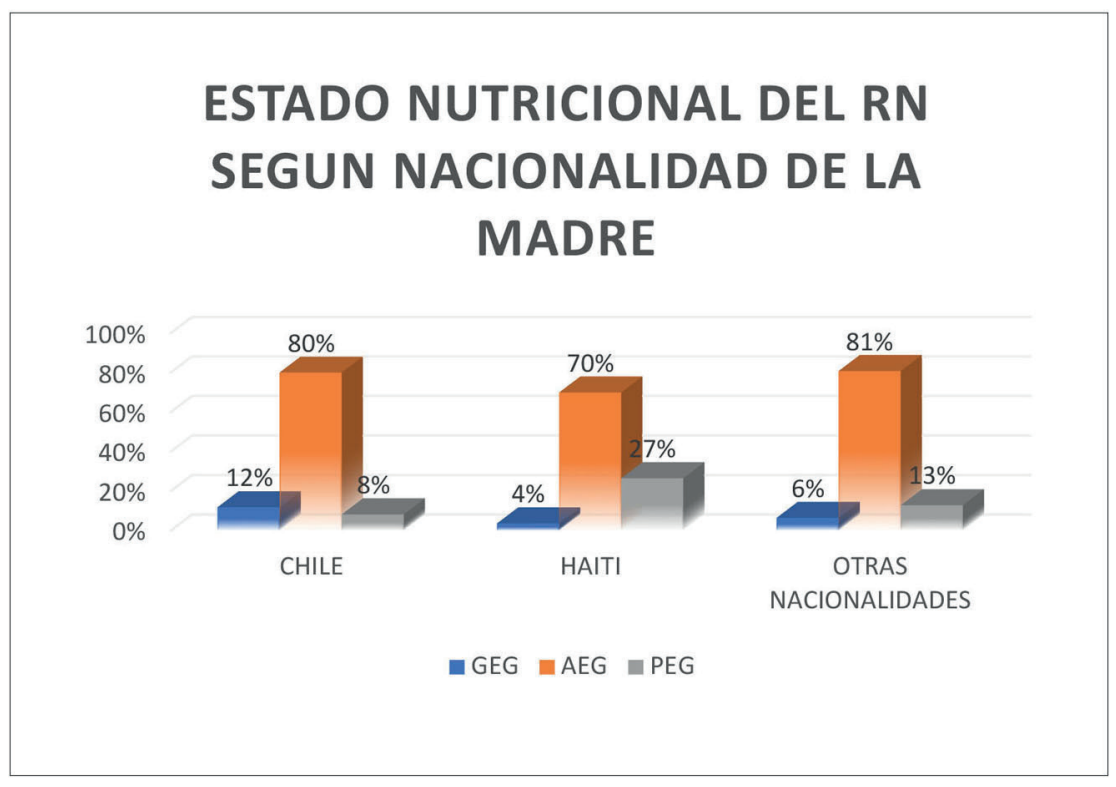

Fuente: Elaboración propia. 
Reyes, S. \& Uarac, M. (2021). Epidemiología neonatal de la migración. UCMaule, 60, enero-junio, 2021, 90-101.

En el periodo estudiado, hubo dos nacimientos gemelares de mujeres haitianas $(2,4 \%)$, dos en mujeres de otras nacionalidades (2,6\%) y 56 nacimientos gemelares en mujeres chilenas $(0,8 \%)$. No es estadísticamente significativo según Test de Fisher.

Debido al gran aumento de nacimientos de hijos de madres haitianas es que decidimos revisar el perfil demográfico de recién nacidos ingresados a la Unidad de Neonatología, lo que se describe a continuación.

\section{Unidad de neonatología (UN)}

El número de ingresos de RN a la Unidad de Neonatología entre los años 2016 y 2018, reflejó un gran aumento de los partos de mujeres haitianas en los últimos 3 años, con una tasa de 0,4 por 1000 nacimientos el año 2016 a 35,5 por 1000 nacimientos el año 2018. Este aumento de partos de mujeres haitianas, genero un aumento de 0 hospitalizaciones el año 2016 a 30 niños hospitalizados, hijos de mujeres haitianas, el año 2018. En la Tabla 2 se detallan las características analizadas según nacionalidad y año estudiado.

Tabla 2: muestra la caracterización de los RN ingresados a la Unidad de Neonatología, según la nacionalidad de la madre en el periodo de 2016 al 2018.

\begin{tabular}{|c|c|c|c|c|c|c|c|c|c|c|}
\hline & \multicolumn{3}{|c|}{2016} & \multicolumn{3}{|c|}{2017} & \multicolumn{3}{|c|}{2018} & \multirow{2}{*}{$\begin{array}{l}\text { SE } \\
p \\
\end{array}$} \\
\hline & CHILE & HAITI & ON & CHILE & HAITI & ON & CHILE & HAITI & ON & \\
\hline INGRESOS & 549 & 0 & 3 & 489 & 1 & 2 & 424 & 30 & 3 & $<0,005$ \\
\hline GEG & 45 & 0 & 0 & 51 & 0 & 1 & 50 & 0 & 0 & \multirow{4}{*}{$<0,025$} \\
\hline AEG & 416 & 0 & 2 & 358 & 0 & 1 & 286 & 19 & 1 & \\
\hline PEG & 36 & 0 & 0 & 35 & 0 & 0 & 50 & 4 & 2 & \\
\hline PEG SEVERO & 51 & 0 & 1 & 44 & 1 & 0 & 38 & 7 & 0 & \\
\hline $\begin{array}{l}\text { Promedio dias } \\
\text { de Hospn }\end{array}$ & 8 & 0 & 4 & 9 & 9 & 7 & 9 & 13,3 & 9,6 & \\
\hline Prematurez & 173 & 0 & 0 & 194 & 0 & 0 & 152 & 6 & 2 & $<0,025$ \\
\hline
\end{tabular}

PEG - pequeño para la edad gestacional; GEG - grande para la edad gestacional; AEG - adecuado para la edad gestacional; SE - estadísticamente significativo; ON - otras nacionalidades; EG - edad gestacional.

Fuente: Elaboración propia.

En el periodo estudiado se observó un descenso en los ingresos totales de RN en la Un, sin embargo, un aumento de los ingresos de hijos de madres haitianas. El porcentaje de hospitalización de hijos de mujeres chilenas fue de un $21 \%$, de mujeres de otras nacionalidades de un $10 \%$ y de mujeres haitianas de un $37 \%$ en el periodo estudiado. Del total de RN de madres haitianas ingresados en el periodo 2016 al 2018 , los principales diagnósticos fueron Pequeño para la Edad Gestacional (PEG) Severo (me- 
Reyes, S. \& Uarac, M. (2021). Epidemiología neonatal de la migración. UCMaule, 60, enero-junio, 2021, 90-101.

nor a percentil 3, según curva de Peso/Edad Gestacional elaborado por Dra. Pittaluga), Hiperbilirrubinemia por incompatibilidad de grupo clásico, Hipoglicemia y Depresión Respiratoria.

Al analizar el RN según EC, se observó una mayor proporción de PEC severo en la población haitiana (26,5\%) versus un $8,9 \%$ del total de RN de origen chileno y $12,8 \%$ de otras nacionalidades (Gráfico 3). Al analizar estadísticamente mediante chi-cuadrado, tiene relación con un $\mathrm{p}<0,025$.

Gráfico 3. Distribución según Estado Nutricional de RN en UN de acuerdo con país origen de la madre.

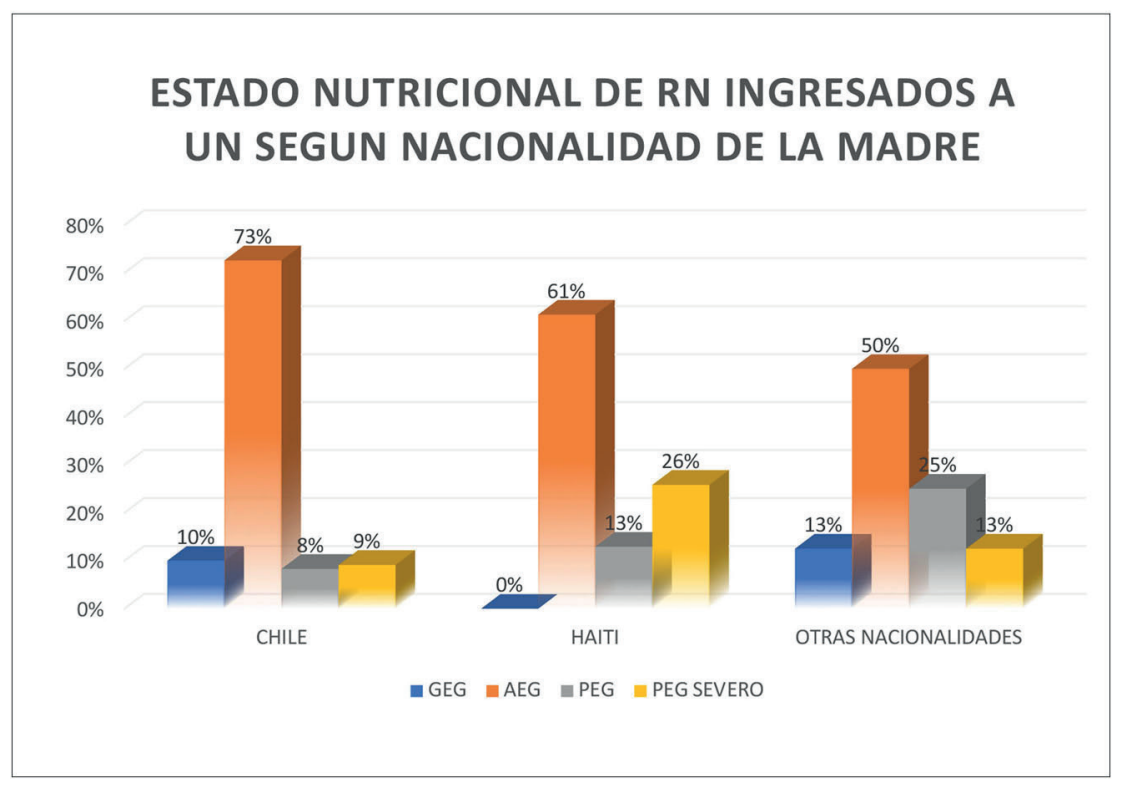

Fuente: Elaboración propia.

Se registró la distribución de edad gestacional del total de RN ingresados a la UN en el periodo estudiado. Los recién nacidos pretérmino (RNPT) (EC $<37$ semanas), hijos de mujeres haitianas corresponde a un 19,4\%, en mujeres de otras nacionalidades a un $25 \%$ y en mujeres chilenas a un $35 \%$ (Tabla 2). Esto es estadísticamente significativo con un $\mathrm{p}<0,025$.

Al revisar la ficha clínica de los pacientes hijos de mujeres haitianas se registró la necesidad de reanimación de estos al momento de nacer, un $20 \%$ requirió alguna maniobra de reanimación con un Apgar promedio al minuto de 5,5 y de 7,6 a los 3 minutos. 
Reyes, S. \& Uarac, M. (2021). Epidemiología neonatal de la migración. UCMaule, 60, enero-junio, 2021, 90-101.

De los pacientes hijos de madres haitianas, en solo 5, la madre presentó antecedentes mórbidos, dato es poco fiable por la barrera lingüística. El promedio de controles prenatales realizadas por las pacientes haitianas fue de 6 en comparación a las chilenas que fue de 7,6 controles, otras nacionalidades, 6,8 controles. Esto no es estadísticamente significativo

Tambien se analizó el tiempo de estadia de los pacientes en la un. Existió un ascenso sostenido del promedio de estadia en la un, de un 7,5 a 8,5 días, durante el periodo estudiado. Llama la atencion el promedio de estadia de los pacientes haitianos (13 días) en relacion a pacientes chilenas 8,6 días y en relacion a hijos de mujeres de otras nacionalidades 6,9 días. Esto no es estadísticamente significativo según $t$ de Student.

\section{DISCUSIÓN}

Según la bibliografía analizada, existe una tendencia a la disminución de la tasa de natalidad en nuestro país (Natalidad General en Chile: 2012-14,0; 2013-13,8; 2014-14,2; 2015-13.6; 2016-12,8 nacimientos/1000 habitantes) y consecuentemente esa misma tendencia en nuestra upp (año 2016-8,9 nacimientos, 2017-8,0 nacimientos y 2018-7,3 nacimientos por 1000 habitantes). Al analizar el lugar de nacimiento de las madres, se ve un notable aumento de los ingresos de mujeres haitianas. Esto se ve reflejado en el porcentaje de los nacimientos de mujeres haitianas en el periodo estudiado: 2016 con un $0,04 \%$ (1) y el 2018 con un 3,6\% (74) del total de ingresos en la upp.

De la misma manera, los ingresos totales a la unidad de neonatología han disminuido, sin embargo, existe un aumento significativo de los hijos de madres haitianas, tal como muestran las cifras con un 0,4/1000 recién nacidos vivos (RNV) en el 2016 a 35,5/1000 RNv en el 2018.

Hemos observado un aumento de los días de hospitalización, lo cual está sustentado en un aumento del promedio de días de estadía de los hijos de madres haitianas (13 días), que supera el promedio general de 8,3 días. Nuestra hipótesis es que la barrera lingüística dificulta la educación a la madre y genera aprehensiones en el personal de salud, con respecto al seguimiento de las indicaciones.

En cuanto a los diagnósticos de ingreso de los pacientes a la un, llama la atención la alta incidencia de PEC severo en hijos de madres haitianas (25,81 \%) versus hijos de madres chilenas $(9,13 \%)$ siendo esto estadísticamente significativo $(p<0,05)$. Por lo anterior, es importante conocer las tablas de crecimiento intrauterino de la población haitiana, la incidencia de patologías maternas que puedan afectar el crecimiento intrauterino y la incidencia de desnutrición en la población haitiana que pueda generar 
una desnutrición transgeneracional en nuestros pacientes. Debe existir una política de salud que amplíe los conocimientos de los funcionarios de la salud, que realizan los controles prenatales y la atención del recién nacido.

Con respecto a la edad gestacional de ingreso de los recién nacidos, se observó un mayor número de RNPT en mujeres chilenas en comparación a mujeres haitianas y de otras nacionalidades, lo cual fue estadísticamente significativo según chi-cuadrado $(p<0,025)$.

Destaca el alto porcentaje de gemelares en la población de origen extranjero (Haití 2,4\%, Otras Nacionalidades 2,6\%) en comparación a la población chilena (0,8\%). Según el Censo del 2017, el año 2009 el porcentaje de nacimientos gemelares en Chile fue de un $1,4 \%$ y el 2011 de $1,91 \%$.

Llama la atención que se produce un mayor porcentaje de hospitalizaciones de los hijos de madres haitianas (37\%) en comparación con las mujeres chilenas (21\%) y de otras nacionalidades (10\%). Esto podría ser secundario a diferencias étnicas que predispone a los pacientes haitianos a presentar una mayor incidencia de patologías perinatales en comparación a la población chilena. Es importante mejorar el conocimiento de nuestros profesionales en relación con las curvas de crecimiento de la descendencia afroamericana, las diferencias culturales en relación con la alimentación y conocer la incidencia de las patologías del embarazo en esta población. Sería útil contar con curvas de crecimiento de pacientes de origen afroamericano dentro de las Guías Clínicas Perinatales (Ministerio de Salud 2015), que permitan realizar una mejor evaluación y diagnóstico. Según la guía perinatal, el Retraso del Crecimiento Intrauterino es una de las complicaciones más frecuentes de la patología obstétrica, que provoca importantes secuelas en la vida adulta, incluyendo Hipertensión Arterial (HTA) y Diabetes Mellitus tipo 2 (DM2). Sus causas pueden ser multifactoriales y es importante determinar, porque en la población haitiana es más frecuente y generar políticas públicas que orienten a mejorar la incidencia de dicha complicación, como por ejemplo, diagnóstico y manejo de HTA en la mujer embarazada.

Es de suma importancia mejorar los registros en el sistema de salud, con el fin de conocer los cambios epidemiológicos que se presentan y que nos exigen adaptarnos a nuevas realidades. Un dato importante, debido al aumento de la inmigración, es la nacionalidad de las pacientes, lo cual no estaba consignado en los registros formales (Ficha de Ingreso a upp, Ficha de Ingreso a un y Ficha Clínica de los RN hospitalizados). Además, es importante que todos los datos solicitados sean registrados en forma fehaciente, debido a que muchas políticas de salud se basan en los registros que entregan las distintas instituciones públicas. 
En relación a lo anterior, el registro de la nacionalidad de las mujeres es importante al considerar que provienen de un país con un sistema de salud menos eficiente, con menor cobertura de inmunizaciones y con enfermedades infectocontagiosas distintas a las nuestras. Siempre y cuando este dato no sea base para discriminación, si no como antecedente que permite dar una mejor atención a las usuarias. Esto significa que debemos hacer un esfuerzo para realizar una educación dirigida a mejorar la cobertura de las inmunizaciones y control de niño sano en esta población, mejorar nuestros conocimientos con respecto a otro tipo de patologías y esforzarnos por superar la barrera lingüística y cultural (Gushulak \& MacPherson, 2004). La llegada de extranjeros al país nos obliga a perfeccionar los programas de vigilancia epidemiológica y capacitar a nuestro personal en el diagnóstico y manejo de patologías poco comunes o desconocidas en la población chilena.

Otro punto importante para considerar son las enfermedades que están en vías de erradicación en Chile, como la tuberculosis (Herrera, 2013) y otras erradicadas como el sarampión, que podrían mostrar un punto de inflexión en el ritmo de decrecimiento, debido a la inmigración de personas provenientes de países con un sistema de salud más precario.

La invitación es a estar dispuestos a conocer eventuales nuevas enfermedades y afrontar el reto de la atención de una nueva sociedad que incluye diferentes etnias, con costumbres y condiciones de salud distintas. 
Reyes, S. \& Uarac, M. (2021). Epidemiología neonatal de la migración. UCMaule, 60, enero-junio, 2021, 90-101. DOI: http://doi.org/10.29035/ucmaule.60.90

\section{REFERENCIAS}

González R., Neira J., Daza P., Nien J., Oyarzún, E., Piedra, D. y Veloso, L. (2019). La paradoja de la inmigración: las madres haitianas y latinoamericanas en Chile. Revista chilena de obstetricia y ginecología, 84(6), 449-459. DOI: https://dx.doi. org/10.4067/S0717-75262019000600449

Gushulak, B. D., \& MacPherson, D. W. (2004). Globalization of infectious diseases: the impact of migration. Clinical infectious diseases: an official publication of the Infectious Diseases Society of America, 38(12), 1742-1748. DOI: https:// doi.org/10.1086/421268

Herrera, T. (2013). La situación de la tuberculosis en Chile y los actuales desafíos: Visita de la OPS al programa de control de la tuberculosis de Chile. Revista chilena de enfermedades respiratorias, 29(1), 46-49. DOI: https://dx.doi.org/10.4067/ S0717-73482013000100008

International Organization for Migration (2020) World Migration Report 2020. Recuperado de: https://publications.iom.int/system/files/pdf/wmr_2020.pdf

Instituto Nacional de Estadísticas. (2018). Características de la inmigración internacional en Chile, Censo 2017. Recuperado de: https://www.censo2017.cl/descargas/inmigracion/181123-documento-migracion.pdf

Instituto Nacional de Estadísticas. (2003). Censo Poblacional 2002: Síntesis de resultados.Recuperado de: https://www.ine.cl/docs/default-source/censo-de-poblacion-y-vivienda/publicaciones-y-anuarios/2002/sintesiscensal-2002.pdf

Milad, A., Novoa, J., Fabres, J., Samamé, M. \& Aspillaga, C. (2010). Recomendación sobre Curvas de Crecimiento Intrauterino. Revista chilena de pediatría, 87(3), 264 274. DOI: https://dx.doi.org/10.4067/S0370-4106201000030001 1

Ministerio de Salud. (2015). Guía Perinatal. Recuperado de: https://www.minsal.cl/sites/ default/files/files/GUIA\%20PERINATAL_2015_\%20PARA\%20PUBLICAR.pdf 